association among persons who were not engaged in strenuous physical activity ( $\mathrm{p}$ for trend $=0.08$ ).

Conclusions Elevated intake of white rice is associated with an increased risk of type 2 diabetes in Japanese women. The finding suggestive of a positive association of rice intake among physically inactive men deserves further investigation.

\section{P2-215 MORTALITY DUE EXCLUSIVELY TO DISEASES FULLY ATTRIBUTABLE TO ALCOHOL CONSUMPTION IN 2006 IN BRAZIL: EFFECTS OF GENDER, EDUCATION AND ETHNICITY}

doi:10.1136/jech.2011.142976j.48

\begin{abstract}
${ }^{1,2}$ Andreia Nascimento. ${ }^{1}$ Institute of Education and Sciences, Hospital Alemão Oswaldo Cruz, São Paulo, Brazil; '2Department of Social Medicine, Faculdade de Ciências Médicas da Santa Casa de São Paulo, São Paulo, Brazil
\end{abstract}

Introduction Diseases fully attributable to alcohol consumption, as mental and behavioural disorders and alcoholic liver disease, are important causes of morbidity and mortality around the world, especially among men. Some studies show higher mortality rates among black people, but in Brazil there are discussions if this finding shows a real association with ethnicity or if socioeconomic status is the leading exposure.

Methods Mortality data and estimated resident population for all Brazilian territory, stratified by age, sex, ethnicity and years of formal education (surrogate for socioeconomic status) were obtained from the Brazilian Mortality Information System (SIM) for 2006. Age-standardised mortality rates were calculated by the direct method using the 2000 Brazilian population.

Results During 2006, 23608 deaths were due exclusively to alcoholattributable diseases in Brazil (2.4\% of total deaths in the country). Men were $89.0 \%$ of subjects. Highest proportion of deaths was observed among people with less than 3 years of formal education. Black men with less than three years of formal education had the highest age-adjusted mortality rate (20.37 deaths/100000 men), followed by black men with more than 3 years of education (11.04). Among white men the age-adjusted mortality rates were 8.43 and 8.57 for these levels of education, respectively. Black women with less formal education showed higher age-adjusted mortality rate (5.82) than black women with more years of formal education (2.59) and white women.

Conclusions Our findings suggest there are important differences in mortality rates related to education and ethnicity for men and women in Brazil.

\section{P2-216 FACTORS ASSOCIATED WITH HOUSEWIVES OBESITY IN IRAN; A NATIONAL SURVEY: STEPS 2005}

\section{doi:10.1136/jech.2011.142976j.49}

${ }^{1} \mathrm{~S}$ Navadeh, ${ }^{2} \mathrm{~L}$ Sajadi, ${ }^{3,4} \mathrm{~A}$ Mirzazadeh, ${ }^{*}{ }^{5} \mathrm{~F}$ Asgari, ${ }^{6} \mathrm{M}$ Haghazali. ${ }^{1}$ Physiology Research Center, Kerman University of Medical Sciences, Kerman, Iran; ${ }^{2}$ Regional Knowledge Hub for HIVIAIDS Surveillance, Kerman University of Medical Sciences, Kerman, Iran; ${ }^{3}$ Department of Epidemiology and Biostatistics, School of Public Health, Tehran University of Medical Sciences, Tehran, Iran; ${ }^{4}$ Physiology Research Center, Kerman University of Medical Sciences, Kerman, Iran; ${ }^{5}$ Center for Disease Control (CDC), The Ministry of Health and Medical Education, Tehran, Iran; ${ }^{6}$ NCD Deputy of Center for Diseases Control, Tehran, Iran

Background We compared obesity risk and its contributors in housewives (HWs) to employees, as a larger portion of women work as housewives in Iran.

Methods Based on the WHO stepwise approach, a cross-sectional survey undertaken throughout Iran in 2005 (33472 women aged 15-65 years) was used to investigate the major risk factors for obesity. Obesity was determined by BMI $>30 \mathrm{kgm}^{-2}$ in adults
(>20 years) and by female BMI percentiles according to WHO 2007 Growth Reference 5-19 years in adolescents ( $<20$ years). We modelled obesity by logistic regression and entered all the known/ potential predictors, including job categories. Weighted prevalence of obesity was calculated by survey analysis series of STATA.

Results The weighted prevalence of overweight and obesity in HWs were $34.5 \%$ and $24.5 \%$ respectively. Employed women were approximately $4 \%$ les overweigh and $10 \%$ less and obese than HWs $(\mathrm{p}<0.01)$. HWs vs employed women had the adjusted OR $1.39(95 \%$ CI 1.18 to 1.63 ) for obesity. Older women, with higher educational level and socioeconomic status, lower physical activities and those living in urban areas were at higher risk of obesity. In comparison to HWs, working as an Official Clerk was associated with a significant decrease $(\mathrm{OR}=0.66)$ odds of obesity, while other employments didn't show a significant association.

Conclusion Working as HW is a significant independent risk factor for obesity in women. Preventive healthcare programs to reduce risk of obesity in women should be targeted by occupation in order to achieve maximum effectiveness.

\section{P2-217 TRENDS IN INCIDENCE AND CASE-FATALITY OF ACUTE MYOCARDIAL INFARCTION IN CHILE, 2001-2007}

doi:10.1136/jech.2011.142976j.50

C Nazzal, ${ }^{*}$ F Alonso. Universidad de Chile, Faculty of Medicine, School of Public Health, Santiago, Chile

Introduction In Chile, coronary heart disease is the main cause of death in men and the second in women. Acute Myocardial Infarction (AMI) causes $73.6 \%$ of coronary heart disease deaths registered in Chile and its incidence is unknown. Aim: to estimate incidence and case-fatality of AMI in Chile and analyse their trends between 2001 and 2007

Methods All cases of AMI (according to the ICD-10, I21 code), registered in the National Hospitalisations and Death databases, were analysed. Annual incidence rates and case-fatality by sex and age groups were calculated. Direct method was used to standardise rates by age, using the WHO 2000 Population. Prais-Winsten regression models were used to evaluate trends, expressed as relative change.

Results Between 2001 and 2007 we estimated that 83754 cases of AMI occurred. Standardised mean annual incidence rate was 74.4 per 100000 inhabitants (98.0 in men and 51.0 in women). Incidence rates increased by $35 \%$ in the age group $<45$ years and $9.2 \%$ in the group of $55-64$ years ( $\mathrm{p}<0.001$, both). Total case fatality was $49.5 \%$ $(45.4 \%$ in men vs $57.2 \%$ in women; $\mathrm{p}<0.001)$. Trend analysis showed a significant annual reduction of $1.2 \%$ in men and $0.81 \%$ in women. In-hospital case fatality was $14.2 \%$, higher in women (11.3\% vs $20.4 \% \mathrm{p}<0.001)$; annual reduction was $0.57 \%$ in men and $1.01 \%$ in women $(p<0.05$ both).

Conclusion AMI incidence was stable, although in younger age groups it increased. Case-fatality decreased both total and inhospital. Despite the greater reduction in women, they still have a higher risk of in-hospital case-fatality.

\section{P2-218 SECONDARY PREVENTION IN ACUTE MYOCARDIAL INFARCTION IN CHILE}

doi:10.1136/jech.2011.142976j.51

${ }^{1} \mathrm{C}$ Nazzal, ${ }^{*} \mathrm{~F}$ Lanas, ${ }^{1} \mathrm{M}$ L Garmendia. ${ }^{1}$ School of Public Health, Faculty of Medicine, Universidad de Chile, Santiago, Chile; ${ }^{2}$ Universidad de la Frontera, Temuco, Chile

Background In 2005 the Chilean government started a healthcare reform that guarantees medical treatment for patients with acute myocardial infarction (MI), including secondary prevention. 